\title{
Dosimetric comparison between VMAT and RC3D techniques: case of prostate treatment
}

\author{
Fatima Zohra Chemingui ${ }^{1, *}$, Fatima Benrachi $^{1}$, Mohamed Saleh Bali $^{2}$, and Hamid Ladjal $^{3}$ \\ ${ }^{1}$ LPMPS Laboratory, Physics Department, Frères Mentouri Constantine1 University, 2500 Constantine, Algeria \\ ${ }^{2}$ Athena Medical Center, 25000 Constantine, Algeria \\ ${ }^{3}$ LIRIS Laboratory, Claude Bernard university, 69100 Lyon, France
}

\begin{abstract}
Considered as the second men cancer in Algeria, prostate cancer is treated in $70 \%$ by radiation. That's why radiation therapy is therapeutic weapon for prostate cancer. Conformational Radiotherapy in $3 \mathrm{D}$ is the most common technique [1-5]. The use of conventionally optimized treatment plans was compared at case scenario of optimized treatment plans VMAT for prostate cancer. The evaluation of the two optimizations strategies focused on the resulting plans ability to retain dose objectives under the influence of patient set up. Dose Volume Histogram in the Planning Target Volume and dose in the Organs At Risks were used to calculate the conformity index, and evaluation ratio of irradiated volume which represent the main tool of comparison [6,7]. The situation was analysed systematically. The $14 \%$ dose increase in the target leads to a decrease in the dose in adjacent organs with $39 \%$ in the bladder. Therefore, the criterion for better efficacy and less toxicity reveal that VMAT is the best choice.
\end{abstract}

\section{Introduction}

Cancer is a term used when abnormal cells divide without control and are able to invade other tissues. In this case, cells can spread to other parts of the body through the blood and lymphatic systems. It is a major topic worldwide. Considered to be responsible for high percentage deaths in Algeria, prostate cancer is the biggest people killer aged between $60-80$ years. The treatment of cancer is summarized in three main categories: drugs, surgery and radiation-therapy. The choice of the treatment depends on the tumor type, location, stage of the disease and general state of patient. Most of time, the treatment is combined. Its ultimate goal is to eradicate the disease, without damaging the surrounding healthy tissues. In practice, the medical decision is always a trade-off and compromise between Tumor Control Probability (TCP) and Normal Tissue Complication Probability (NTCP) [3, 5, 8-10].

At present, radiation therapy (RT) is used in about $70 \%$ of all cancer treatments in industrialized countries. It allows killing tumor cells, by deposing a high dose of radiation within the tumor. In North Africa and exactly in Algeria the first private Anti Cancer Center "Athena Medical Center", treating with conventional techniques, was opened in 2013. The evolution of photon delivery techniques using conventional linear accelerator equipped with multi-leaf collimator as RC3D Conformational or VMAT [11-15]. The main task of a medical physicist is to make sure that the right dose is delivered in the right place. Treatment planning systems (TPS) was used to plan radio therapy treatments, but with limited dose accuracy in some particular cases. On the other hand, the OAR must be conserved and receive the minimum of dose. In prostate cancer, the better ballistic of VMAT over RC3D allows decreasing the dose to surrounding tissues and achieves through 76 Gy in PTV.

\section{Material and method}

Treatment planning (TP) is the function that translates the physician's prescription and intent into a treatment plan. It contains the set of equipment parameters that control the dose deposition in patient expected to be equivalent to the treatment plan predicted dose. This definition often implies many requirements often above and beyond those implied for photon TP. For the optimization, the physician's prescription is a set of quantified statements (i.e., maximum dose to target), suitable for numeric manipulation in an optimization algorithm, and generalized intent (i.e., minimize dose to the OAR). The quality of a particular objective is limited by the prescription that fixes the boundaries of what remains possible in a trade-off consideration of competing objectives $[11,16,17]$.

Corresponding author: z.chemingui@hotmail.fr 
In this context, two patients with curator treatment were the subject of this retrospective study. They were selected for stage and volume. The treatment parameters for a chosen patient are listed in Table 1. The two patients have undergone 3DCT simulation using immobilization device (headrest, footrest, foot wedge medical solutions) with General Electric Optima Radiation Therapy CT scanner. Scans covered the entire pelvis volume and upper abdomen part. Then, CT data imaging were generated using the treatment planning system Eclipse (soma version 11.3 by Varian Medical System, California, USA). Gross tumor volume (GTV) was contoured by the treating radiation oncologist from CT datasets. For each prostate cancer case two treatment plans were generated accounting for treatment uncertainties in two different ways:

VMAT plans were developed using the Eclipse (Version 11.3 Varian Medical System, California, USA) Treatment Planning System (TPS) with 6 and $18 \mathrm{MV}$ for each patient. AAA (Analytical Anisotropic Algorithm, Varian Medical System, California, USA) was used to compute the dose distributions. Inverse pelvis volume and upper abdomen part. Then, CT data imaging were generated using the treatment planning system Eclipse (soma version 11.3 by Varian Medical System, California, USA). Gross tumor volume (GTV) was contoured by the treating radiation oncologist from CT datasets. For each prostate cancer case two treatment plans were generated accounting for treatment uncertainties in two different ways:

VMAT plans were developed using the Eclipse (Version 11.3 Varian Medical System, California, USA) TPS with 6 and $18 \mathrm{MV}$ for each patient. AAA (Analytical Anisotropic Algorithm, Varian Medical System, California, USA) was used to compute the dose distributions. Inverse treatment plans for VMAT were

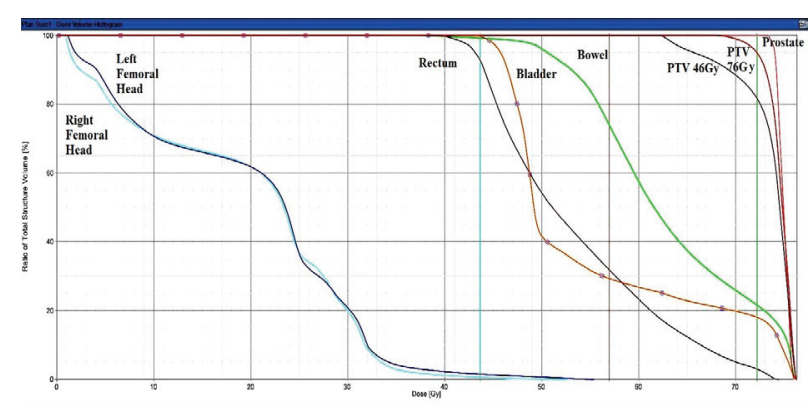

generated using the same dose-volume constraints for all plans. The dose constraints was set for the rectal wall, rectum, bladder, bowel, femoral heads and unspecified normal structure.

For the RC3D radiotherapy planning, after contouring all normal structures and critical organ, 7 fields treatment techniques were used for the smallest volume PTV $76 \mathrm{~Gy}$. Beam arrangements $0^{\circ}, 45^{\circ}, 90^{\circ}$, $135^{\circ}, 225^{\circ}, 270^{\circ}, 315^{\circ}$ angles or orientations were used. The prescribed dose was normalized to $100 \%$ at the isocenter, and 95\% isodose surface covered the PTV.

Dose Volume Histograms (DVHs) were used to compare treatment plans including PTV, OAR, between two treatment techniques. Then, doses distributions were evaluated and in particular points of DVHs [12] by conformity index (CI) defined by tumoral volume covered by reference Isodose, $(\mathrm{CI}$, ideal value $=1)$,

$$
C I=\frac{V_{I R}}{V_{T}}
$$

$\mathrm{V}_{\mathrm{IR}}$ and $\mathrm{V}_{\mathrm{T}}$ denote respectively volume of reference Isodose, and Tumoral Volume.

\section{Results and discussion}

Fig 1. shows DVHs curves of dose distribution in prostatic irradiation with $76 \mathrm{~Gy}$.

Dose distribution illustrated by DVHs curves for patient treated for prostate cancer: (Right) VMAT plan, (left) RC3D plan. The PTV 76Gy is represented in red, in Dark brown: PTV 46Gy, in Blue: left femoral head, in Cyan: Right Femoral head, in Green: Bowel, in Brown: Rectum, Orange: Bladder, Pink: Prostate.

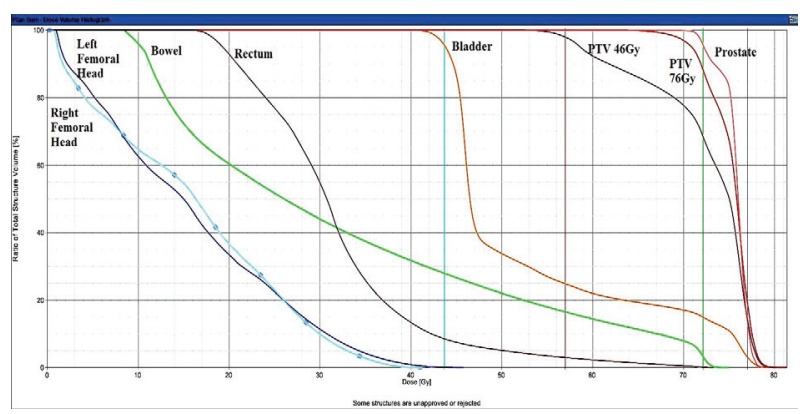

Fig. 1. DVHs: Comparison of dose distribution between VMAT and RC3D

Dose differences between VMAT and RC3D plans in different structures of OAR (rectum wall, rectum, bladder, bowel, and femoral heads) are illustrated in Table 1. This observational study shows DVHs Curves of prostatic irradiation at 76Gy, for VMAT and RC3D plans. The mean DVH are obtained in OARs, which are mentioned above. The DVHs curves obtained by VMAT are underneath those obtained by RC3D. For the other structures; bladder and soft tissues, the curves cross each other in several times. We can observe that the mean dose in different OARs is significantly reduced in VMAT plans. The values of this reduction attend their maximum for the bladder with more than $40 \%$ of reduction in the mean dose. 
Table 1. Difference in the delivered mean dose and maximum dose between the RC3D plan and the VMAT plan for PTVs and OARs

\begin{tabular}{|l|c|c|c|}
\hline Organs & Volume $\left(\mathbf{c m}^{\mathbf{3}}\right)$ & $\Delta$ Dose $\mathbf{m a x}(\mathbf{G y})$ & $\Delta$ Dose mean $(\mathbf{G y})$ \\
\hline Rectum & 74 & $-1,132$ & $-21,597$ \\
\hline Rectal wall & 30,2 & $-6,771$ & $-23,422$ \\
\hline Bladder & 142,8 & $-1,230$ & $-30,797$ \\
\hline Bowel & 1094,7 & $-4,455$ & $-4,646$ \\
\hline FBR & 174,6 & $-9,619$ & $-4,343$ \\
\hline FBL & 181,8 & $-11,508$ & $-3,792$ \\
\hline PTV 60Gy & 190,1 & 5,056 & 21,085 \\
\hline PTV 76Gy & 141,4 & 5,056 & 0,643 \\
\hline
\end{tabular}

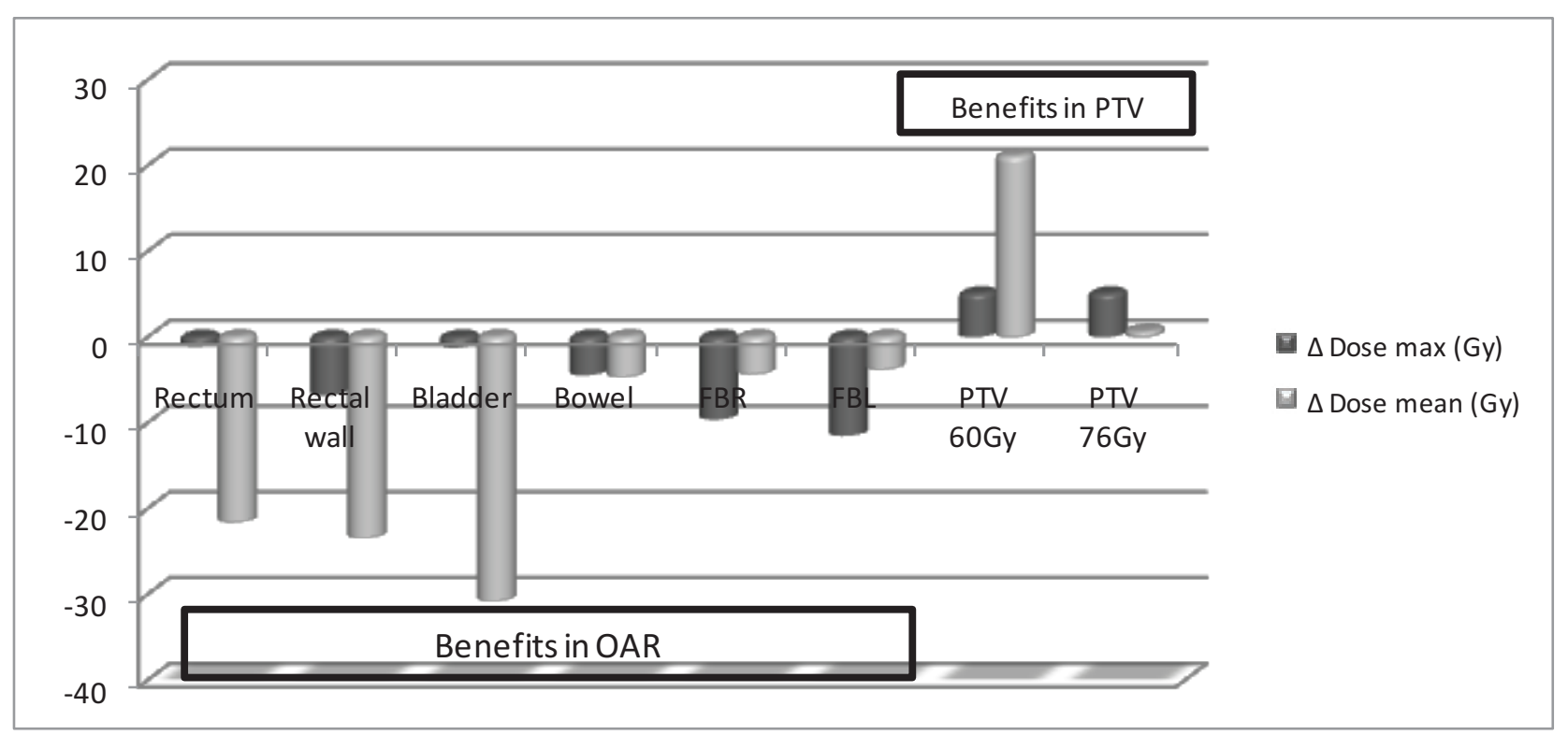

Fig. 2. Graphical representation of $\Delta$ dose means and max between the VMAT and RC3D plans

Table 2. Comparison between Conformity indexes of VMAT and 3DRC plans

\begin{tabular}{|l|c|c|c|}
\cline { 2 - 4 } \multicolumn{1}{c|}{} & $\begin{array}{c}\text { CI RC3D ISODOSE } \\
\text { 95\% 76GY (72.2) }\end{array}$ & $\begin{array}{c}\text { CI VMAT ISODOSE } \\
\text { 95\% 76GY (72.2) }\end{array}$ & Diff CI \\
\hline Patient 1 & 0.7312 & 1.0855 & 0.3543 \\
\hline Patient 2 & 0.7956 & 0.9306 & 0.135 \\
\hline
\end{tabular}

In the other hand, we can see in Table 2 the difference in term of CI between VMAT and RC3D plans. The values of CI for the RC3D plans are less than 0.8 otherwise VMAT plan values are upper than 0.9 .

Dose values (maxima and mean) of particular PTV 46 Gy, PTV 60 Gy, PTV 76 Gy and OARs are exposed, and we can observe that the mean dose in rectum decreases by about $30 \%$ with VMAT technique. In volume covered by dose of 60 Gy (V60) and 70 Gy (V70), it decreases respectively by $20 \%, 14 \%$. For bladder, it decreases by $10 \%$ and $18 \%$ respectively. For femoral heads, VMAT dose decrease in V30 from $20 \%$ to $9 \%$. Table 2 shows VMAT technique conformity index values, varied from 0.93 to 1.0 and are all close to the ideal value otherwise for RC3D technique.

To help the analysis, we affected some statistical tests, and we calculated the VMAT improvement ratio of conformation in PTVs. the results are represented in Fig. 3.

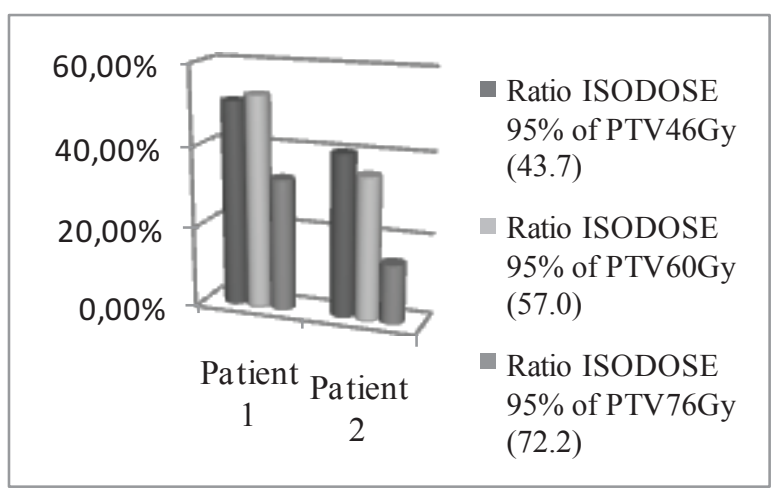

Fig. 3. Reduction of the $95 \%$ isodose volume in the VMAT plans compared to the RC3D plans.

The PTV 46 Gy volume is ameliorated by factor of $50 \%$ due to volume containing all OARs. For PTV 60 Gy, the improvement is more than $30 \%$, this volume is smaller than PTV 46 Gy it contains rectum and bladder. 
For PTV $76 \mathrm{~Gy}$, the value of this improvement is more than $14 \%$ and consists prostate only.

\section{Conclusion}

In this study, we evaluated the dose distribution plans obtained from two techniques RC3D and VMAT. The VMAT plans gave more effectiveness in treated volumes by increasing the dose mean in PTVs corresponding to the prostate tumor and the nodal regions. VMAT technique offers best conformation in target volumes (PTVs) and less toxicity in soft tissues (less dose in OARs). Actually, in developed countries VMAT have replaced $\mathrm{RC} 3 \mathrm{D}$ in the treatment of prostate cancer. In Algeria, Athena Medical Center is the only cancer center offering this technique in clinical routine.

\section{References}

1. F. Alongi, et al., Rad. Oncol. 8, 171 (2013)
2. D. Wolff et al., Med. Phys. German. 19, 58 (2009)

3. M. M. Aspradakis et al., Phys. Med. Biol. 48, 2873 (2003)

4. R. Garcia, Validations dosimétriques des conditions cliniques des radiothérapies thoraciques, (Ph. D Thesis, Université Toulouse III - Paul Sabatier 2013)

5. ICRU Report 78, J. ICRU 7, (2007)

6. C. Popescu et al., Int. J. Rad. Onco. Biol. Phys. 76, 287 (2009)

7. J. Zhu, Modèles prédictifs de toxicité en radiothérapie par modulation d'intensité, (Ph. D Thesis, Université de Rennes I. 2013)

8. ESTRO booklet $\mathrm{n}$ 09: Guidelines for the verification of IMRT (2008)

9. A. Pollack et al., Rad. Onco. Biol. Phys. 53, 1097 (2002)

10. H. M. Kooy et al., Philadelphia, Lippincott. Williams. \& Wilkins. 70, 70 (2008)

11. D. M. Hall et al., Rad. Onco. 10, 115 (2016)

12. J. He et al., J. Thorac. Dis. 8, 862 (2016)

13. A. Cakir et al., Asian. Pac. J. Cancer. Prev. 16, 3365 (2015)

14. C. Vargas, Int. J. Rad. Onco. Biol. Phys. 62, 1297 (2005)

15. S.J. Feigenberg et al., Int. J. Onco. Biol. Phys. 62, 397 (2005)

16. M. Valeriani et al., Biol. Med. Res. Int. 10, 6 (2014)

17. P.A. Kupelian et al., Int J Rad. Onco. Biol. Phys. 58, 25 (2004) 